\title{
CLINICAL STUDY OF ELECTROCARDIOGRAM CHANGES IN DIFFERENT TYPES OF CEREBROVASCULAR ACCIDENTS AND PROGNOSTIC SIGNIFICANCE
}

\author{
Parashuram¹, Srinivasa Kaligonahalli Venkataramanappa²
}

${ }_{1}^{1}$ Assistant Professor, Department of Medicine, Dr. B. R. Ambedkar Medical College and Hospital, Bangalore. ${ }^{2}$ Associate Professor, Department of Medicine, Dr. B. R. Ambedkar Medical College and Hospital, Bangalore.

ABSTRACT

\section{BACKGROUND AND OBJECTIVES}

Stroke is one of the most common causes of death worldwide. People who suffer cerebrovascular accidents also have an abnormality in the hearts electrical cycle are at a higher risk of death with 90 days than people who do not have abnormal electrical activity. The ECG changes have been reported predominately with subarachnoid haemorrhage and intracerebral haemorrhage and in a few percentage of cases of cerebral infarct. Present study is an attempt to study the pattern ECG abnormalities in patients with cerebrovascular accidents and its prognostic significance.

\section{METHODOLOGY}

Prospective study of 100 patients admitted to the casualty or outpatient department of B. R. Ambedkar Medical Hospital, K. G. Halli, Bangalore, with diagnosis of first episode of cerebrovascular accident were taken up for the study.

\section{RESULT}

Among 100 patients, 59 were males and 41 were females. The incidence of stroke increases with age $22 \%$ between 60 and 65 days to $40 \%$ above 70 years. Cerebral thrombosis was the most common cerebrovascular accident seen in 53 patients and subarachnoid haemorrhage least common seen in six patients. The ECG changes were seen in 82 patients. The ECG changes were QTC prolongation (30\%), ST segment changes (28\%). T-wave changes (44\%), U-wave changes (10\%), LVH (36\%), sinus bradycardia (10\%), sinus tachycardia (24\%). These abnormalities were commonly seen in cerebral haemorrhage and subarachnoid haemorrhage than the infarct. The overall immediate mortality was $22 \%$ and did not relate to the ECG changes seen, but was dependent on level of consciousness, type of CVA, extent of lesion and other co-existent disease.

\section{CONCLUSION}

Patient with cerebrovascular accident often have abnormal ECG changes in absence of unknown organic heart disease or electrolyte imbalance, more common in intracerebral haemorrhage and subarachnoid haemorrhage. The most common ECG changes are T-wave changes, prolonged QTC, ST segment changes and arrhythmias. The ECG changes did not relate to the mortality in patients with cerebrovascular accidents.

\section{KEYWORDS}

Cerebrovascular Accidents, ECG Changes, T-Wave Changes, Intracerebral Haemorrhage.

HOW TO CITE THIS ARTICLE: Parashuram, Venkataramanappa SK. Clinical study of electrocardiogram changes in different types of cerebrovascular accidents and prognostic significance. J. Evolution Med. Dent. Sci. 2016;5(49):3127-3131, DOI: $10.14260 /$ jemds/2016/726

\section{INTRODUCTION}

Stroke is currently the second leading cause of death in the Western world, ranking after heart disease and before cancer, and causes $10 \%$ of deaths worldwide. People who suffer an ischaemic stroke and also have an abnormality in the heart's electrical cycle are at a higher risk of death within 90 days than people who do not have abnormal electrical activity at the time of emergency treatment according to new research. Electrocardiographic (ECG) abnormalities have been observed in acute cerebrovascular events; ECG abnormalities are frequent in acute stroke and may predict 6-month mortality.

Abnormalities of the electrocardiogram are extremely useful in the recognition of heart disease, but they also occur in a variety of states in which the primary pathology is

Financial or Other, Competing Interest: None.

Submission 24-05-2016, Peer Review 31-05-2016,

Acceptance 03-06-2016, Published 17-06-2016.

Corresponding Author:

Dr. Parashuram,

Assistant Professor,

Department of Medicine,

Dr. B. R. Ambedkar Medical College and Hospital,

Bangalore.

E-mail:drbhavimani@gmail.com

DOI: 10.14260/jemds/2016/726 extracardiac. Among these, neurological disease is of special interest. The abnormalities which sometimes occur in neurological diseases are among the most striking deviation from normal that have been observed. In addition, neurogenic changes in the electrocardiogram furnish valuable insights into the physiologic basis of this record. Like other electrocardiographic abnormalities with an extracardiac basis, and those of neurogenic origin should not be taken as evidence of primary heart disease.

\section{The Present Study Aims}

To study the electrocardiographic changes observed in different types of cerebrovascular accident. To study the prevalence of ECG changes in different types of cerebrovascular accident. To establish the prognostic significance of the ECG changes.

\section{MATERIALS AND METHODS}

The material for the present prospective study of 100 subjects admitted to casualty/OPD from September 2008 to January 2011 of Dr. B.R. Ambedkar Medical College Hospital, K. G. Halli, Bangalore.

With the diagnosis of cerebrovascular accidents age group of above 60 years, the cases studied were prospective detailed clinical history was recorded with particular 
reference to the central nervous system and the cardiovascular system. Care was focused on eliminating any patient from the study with evidence of previous ischaemic heart disease, congenital, valvular or cardiomyopathic heart disease. However, patients with hypertension/diabetes mellitus were included in the study. Electrolyte, profiles were a requisite and all patients with electrolyte imbalance excluded from the study; 100 subjects with CVA who had no evidence of prior cardiac abnormalities, no prior history of stroke or reasons for electrolyte effect abnormalities on their electrocardiograms were included.

A thorough physical examination was done with special emphasis to the nervous system and cardiovascular system examination. Examination also included vital signs, especially blood pressure changes, peripheral pulses including the carotid and evidence of atherosclerosis.

\section{Investigations Done Included}

- Complete blood picture.

- Urine examination.

- ECG

- Blood urea, Serum Creatinine.

- Serum Electrolytes.

- Lipid Profile.

- Chest X-ray.

Cardiac enzymes were done in some patients where there was a suspicion of myocardial infarction. Serum VDRL was done in relevant cases. CT scan of the head was done in all cases. Lumbar puncture was done only in those cases, where it was essential for diagnosis; 2D echocardiograms were done in a few cases where there was suspicion of heart diseases.

- 12 lead ECG was taken for all cases as soon as the patient was admitted.

- A repeat ECG was done 3-5 days later in all patients who survived.

Patients were assigned to one of the following types of cerebrovascular accidents.

- Cerebral thrombosis.

- Cerebral embolism.

- Cerebral haemorrhage.

- Subarachnoid haemorrhage.

In Analysing the ECG, the following Criteria were considered to Recognise the Abnormality

\section{Sinus Arrhythmia}

This was considered when there were alternating periods of gradually lengthening and gradually shortening P-P intervals with a normal P-wave and PR interval.

\section{Sinus Tachycardia}

This was considered when the heart rate exceeded 100/min.

\section{Sinus Bradycardia}

This was considered when the heart rate was less than $60 / \mathrm{min}$.

\section{P-Pulmonale}

They are tall peaked $\mathrm{P}$ waves greater than $2.5 \mathrm{~mm}$ seen in leads II, III, AVF.

\section{P-Mitrale}

Bifid P $>0.10$ second in lead II and I or P-terminal force in V1 exceeding $0.03 \mathrm{~mm}$.

\section{Q Waves}

- They were considered significant (Unless confined to lead III) if they were greater than,

- $0.04 \mathrm{sec}$ in duration and $1 / 4$ of the R-wave for the lead.

\section{ST Segment}

ST segment elevation of more than $1 \mathrm{~mm}$ or depression more than $0.5 \mathrm{~mm}$ (In the absence of digoxin treatment) was taken abnormal.

\section{T-Wave}

The T-wave abnormality was considered when there was inversion of $\mathrm{T}$ waves, flat $\mathrm{T}$ waves or abnormal tall $\mathrm{T}$ waves of $10 \mathrm{~mm}$ in chest leads and mid-precordial leads (Exceeding $60 \%$ of the height of R-wave.

\section{U-Wave}

This was considered significant when exaggeration of U-wave voltage was noted when appeared in more than 2 leads. When appeared in leads, in which it was not normally seen (V2-V4).

\section{INVERTED ‘U’ WAVES \\ Q-Tc Interval}

QT interval was measured and corrected for the heart rate. The normal Q-Tc is $0.35 \mathrm{sec}$ to $0.43 \mathrm{sec}$. It is measured by following formula and expressed in seconds.

\section{LEFT VENTRICULAR HYPERTROPHY}

It is considered according to Romhilt and Estes Point Score System. As follows.
Increased QRS magnitude

ST-T abnormalities

ASP wave of left atrial enlargement

Left axis deviation

$=3$ points.

$=3$ points.

$=3$ points.

$=2$ points.

Increased ventricular activation time $=1$ points.
A score of 5 points or more indicates left increased ventricular hypertrophy and correlates with increased ventricular mass.

\section{Right Ventricular Hypertrophy}

- R-wave voltage was greater than $5 \mathrm{~mm}$ in V1 with ST segment depression and T-wave inversion in V1-V3.

- Persistent S-wave in V5-V6 clock wise electrical rotation. Right axis deviation.

- A delay in the inscription of the intrinsicoid deflexion in right oriented leads.

- $\quad$ RBBB was identified by RSR' complexes in V1-V2, Wide S-wave in V5-V6 and standard lead I QRS interval $>0.12$ sec.

- $\quad$ ST depression and 'T' wave inversion in V1-V3 LBBB was identified by wide slurred R-waves in V4-V6 with absent Q-wave in these leads. Slurred S-wave in V1 QRS interval $>0.16$ sec. ST depression and T-wave inversion in V4-V6.

\section{RESULTS}

\section{Observation and Analysis}

Hundred patients admitted with stroke were studied in detail and following observations were noted. 


\begin{tabular}{|c|c|c|c|}
\hline Age Group & Male & Female & Total \\
\hline $60-65$ yrs. & 16 & 10 & 26 \\
\hline $65-70$ & 20 & 14 & 34 \\
\hline$>70$ & 23 & 17 & 40 \\
\hline Total & $\mathbf{5 9}$ & $\mathbf{4 1}$ & $\mathbf{1 0 0}$ \\
\hline \multicolumn{4}{|c|}{ Table I: Age and Sex Distribution } \\
\hline
\end{tabular}

The cerebrovascular accidents increase with age with $26 \%$ in the age group of $60-65$ and $40 \%$ above 70 yrs. The youngest patient with CVA was 60 yrs. and the eldest was 86 yrs. old.

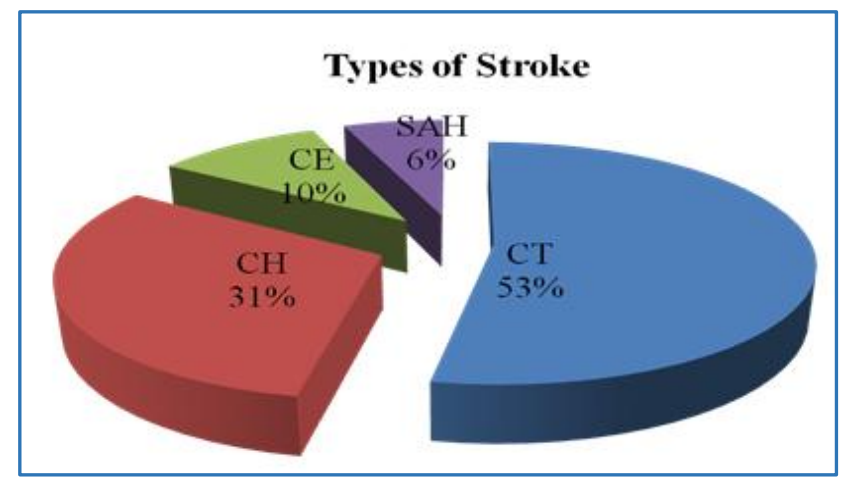

Graphical Representation of Type of Stroke

\begin{tabular}{|c|c|c|c|c|c|c|}
\hline & CT & CH & SAH & CE & Total & \% \\
\hline Q-Tc Prolongation & 14 & 14 & 0 & 2 & 30 & 30 \\
\hline ST Segment Changes & 6 & 16 & 4 & 2 & 28 & 28 \\
\hline T-Wave Changes & 10 & 22 & 6 & 6 & 44 & 44 \\
\hline U-Waves & 0 & 6 & 4 & 0 & 10 & 10 \\
\hline Q-Waves & 2 & 0 & 0 & 0 & 2 & 4 \\
\hline LVH & 16 & 18 & 2 & 0 & 36 & 36 \\
\hline Sinus Arrhythmia & 0 & 2 & 0 & 0 & 2 & 2 \\
\hline Sinus Tachycardia & 10 & 10 & 2 & 2 & 24 & 24 \\
\hline Sinus Bradycardia & 6 & 4 & 0 & 0 & 10 & 10 \\
\hline Atrial Fibrillation & 2 & 0 & 0 & 2 & 4 & 4 \\
\hline Vent. Premature & 2 & 0 & 0 & 0 & 2 & 2 \\
\hline Beats & 0 & 2 & 0 & 0 & 2 & 2 \\
\hline LBBB & 2 & 0 & 0 & 0 & 2 & 2 \\
\hline RBBB & 0 & 4 & 2 & 0 & 6 & 6 \\
\hline P pulmonale & 13 & 1 & 1 & 3 & 18 & 18 \\
\hline Normal & Table II: ECG Findings in Cerebrovascular Accidents showing \\
\hline \multicolumn{7}{|c|}{ Typecific ECG Changes in Different } \\
\hline
\end{tabular}

The above table lists the various ECG findings seen in different types of strokes. The most common ECG abnormality associated with stroke was T-wave changes (44\%).

Followed by left ventricular hypertrophy pattern with or without strain (36\%), prolonged Q-Tc interval.

$(30 \%)$ ST segment changes $(28 \%)$, sinus tachycardia (24\%), sinus bradycardia (10\%) and U waves (10\%).

\section{T-Wave Changes}

$44 \%$ of patients showed T-wave changes and these included 24 cases of inverted $T$ waves, 8 cases with flat $T$ waves, and 12 cases of biphasic $\mathrm{T}$ waves.

And 4 cases of tall T waves. In some cases where EGG was repeated after a few days, $\mathrm{T}$-wave reverted to normal. T-wave changes were more frequent in cerebral haemorrhage.
Left Ventricular Hypertrophy Pattern

$36 \%$ of patients showed left ventricular hypertrophy pattern (By voltage criteria) with or without strain pattern. All of them were hypertensive.

\section{Q-Tc Prolongation}

Q-Tc was prolonged in $30 \%$ of patients. It occurred more frequently in patients with intracranial haemorrhage (i.e. 7 out of the 17 patients $=41 \%$ ) and cerebral thrombosis (i.e. 7 out of 21 patients $=33 \%$ ).

\section{ST Segment Changes}

ST segment changes were seen in $28 \%$ of patients (i.e. 14 out of 50 patients); 10 of them had ST segment depression and 4 of them had ST segment elevation.

ST segment changes were more frequent in cerebral haemorrhage (8 out of 17 patients, i.e. $47 \%$ ) and subarachnoid haemorrhage ( 2 out of 6 patients, i.e. 33\%). One of the cases (a case of cerebral haemorrhage) where the EGG showed ST segment elevation (In leads V1-V4) developed acute anterior wall myocardial infarction.

\section{Arrhythmias}

Among the arrhythmias, sinus tachycardia was seen in $24 \%$ of cases, sinus bradycardia in $10 \%$ cases. Atrial fibrillation in $4 \%$ cases and sinus arrhythmia in $2 \%$ of cases.

\section{U Waves}

U-wave were seen in $10 \%$ of cases. They occurred more frequently in patients with cerebral haemorrhage and subarachnoid haemorrhage (18\% and 33\%, respectively).

\section{Other Abnormalities}

Other abnormalities that were seen in EGG were P. Pulmonale $(6 \%)$, ventricular premature beats $(2 \%)$, right bundle branch block (2\%), left bundle branch fascicular block (2\%) and Q waves $(2 \%)$.

\begin{tabular}{|c|c|c|c|}
\hline $\begin{array}{c}\text { Types of } \\
\text { CVA }\end{array}$ & $\begin{array}{c}\text { Total No. of } \\
\text { Patients }\end{array}$ & $\begin{array}{c}\text { No. of } \\
\text { Patients } \\
\text { Expired }\end{array}$ & $\mathbf{\%}$ \\
\hline $\begin{array}{c}\text { Cerebral } \\
\text { thrombosis }\end{array}$ & 53 & 2 & 3.7 \\
\hline $\begin{array}{c}\text { Cerebral } \\
\text { haemorrhage }\end{array}$ & 31 & 16 & 51.6 \\
\hline $\begin{array}{c}\text { Subarachnoid } \\
\text { haemorrhage }\end{array}$ & 6 & 2 & 33.34 \\
\hline Cerebral embolism & 10 & 2 & 20 \\
\hline Total & $\mathbf{1 0 0}$ & $\mathbf{2 2}$ & $\mathbf{2 2}$ \\
\hline $\begin{array}{c}\text { Table III: Mortality in Stroke showing Immediate Mortality } \\
\text { in Different Types of CVA }\end{array}$ \\
\hline
\end{tabular}

Overall immediate mortality was $22 \%$. It was high in cerebral haemorrhage $(51.6 \%)$ followed by subarachnoid haemorrhage (22.34) and cerebral embolism (20\%); $3.7 \%$ of patients with cerebral thrombosis expired.

\begin{tabular}{|c|c|c|c|}
\hline $\begin{array}{c}\text { Level of } \\
\text { Consciousness }\end{array}$ & $\begin{array}{c}\text { Total No. of } \\
\text { Patients }\end{array}$ & $\begin{array}{c}\text { No. of } \\
\text { Patients } \\
\text { Expired }\end{array}$ & \% \\
\hline Alert & 2 & 38 & 5.26 \\
\hline Drowsy & 10 & 42 & 23.8 \\
\hline Stuporous/Comatose & 10 & 20 & 50 \\
\hline Total & $\mathbf{2 2}$ & $\mathbf{1 0 0}$ & $\mathbf{2 2}$ \\
\hline Table IV: Showing Relation of Level of Consciousness to \\
Immediate Mortality \\
\hline
\end{tabular}


As evident from the table, the highest mortality was seen in comatose patients (50\%) and least in alert patients (5.26\%).

An attempt was made to correlate the mortality in these patients with the ECG changes.

It was found that the mortality in these patients did not relate to the ECG changes seen, but was dependent on the level of consciousness on admission, the type of cerebrovascular accident, the extent of the lesion and other co-existent disease.

\section{DISCUSSION}

Abnormal electrocardiograms occurring with acute cerebrovascular events have been previously described by many authors. The incidence of EGG changes is higher with intracerebral haemorrhage and subarachnoid haemorrhage than with cerebral infarct. ${ }^{1}$

Kaya, McDonald and Randall have demonstrated subendocardial haemorrhages after prolonged and intense sympathetic activity on the heart, such a condition of intense sympathetic drive may occur during an acute cerebrovascular accident. However, acute myocardial damage is unusual. ${ }^{2}$

Direct stimulation of various areas of the brain is known to result in abnormal electrical pattern of the heart. Gropp and Manning have suggested that lesion of area 13 on the orbital surface of the frontal lobe may be responsible for the ECG changes. It is therefore proposed that the cause of ECG abnormalities in association with lesions in the vicinity of area 13 on the orbital surface of the frontal lobe or around the circle of Willis results from alteration in sympathetic and parasympathetic tone mediated by fibres from the orbitofrontal area to the heart via the stellate ganglion. In the present study, all the 50 patients with stroke were studied for ECG changes on admission. Some of the cases had serial ECG's done. The changes observed were attributed to stroke after ruling out other possible factors like electrolyte disturbances. In the present study, 18\% had normal ECG and the remaining $82 \%$ showed some ECG changes. Q-Tc prolongation which comprised $30 \%$ in the present study was reported as a frequent finding in studies by Goldstein (1979), Fentz (1962), Mathur (1966) Mayo Clinic (2009) etc.

Goldstein 1979 ECG findings were normal 8, abnormal 92, QTc prolongations 45 , T waves 29 , U waves 28 , ST changes 27 , sinus tachycardia 28, LVH 26, Sinus bradycardia 7, Atrial fibrillation 14, Q waves 20. This was found to be more frequent in patients with intracranial haemorrhage (41\%) compared to other types of stroke. Similar observations were also described by Goldstein. ${ }^{3}$

$\mathrm{T}$-wave changes in 20 of cases in comparison to $44 \%$ of cases in the present study. T-wave changes were more frequent in cerebral haemorrhage. Similar T-wave changes were described by Fentz (1962), Mathur (1966), Ananthachari (1967), Fure B (2006), etc. U-wave seen in 10\% of cases in comparison to $28 \%$ of cases in the study done by Goldstein. They were more common in subarachnoid haemorrhage (33\%) and intracerebral haemorrhage (18\%). ST depression was found in $20 \%$ of cases against $27 \%$ of cases in the study done by Goldstein. The other findings seen in the present study has been described by various authors in variable frequency.

Negh KH (2004), the ECG rhythms and ischaemic changes of 97 elderly patients admitted with acute stroke or Transient
Ischemic Attack (TIA) were compared with those of 70 medical controls admitted during the same study period. Patient's median age was 80 years. Atrial fibrillation occurred in 26 stroke/TIA patients $(27 \%)$ and 17 control patients (24\%). Ischaemic ECG changes occurred in 54 stroke/TIA patients (56\%) and 32 control patients (46\%).

Fure B (2006), the aims of the present study were to assess the prevalence of ECG changes in acute ischaemic stroke, a total of 279 patients suffering from acute ischaemic stroke were included prospectively in the study. The most frequent ECG changes were prolonged QTc 36.0\%, ST depression $24.5 \%$, atrial fibrillation $19.9 \%$ and $\mathrm{T}$-wave inversion $17.8 \%$.

In the present study findings are 100 patients, among them 59 were male and 41 were female. The incidence of stroke in the age group below 45 years was $22 \%$ and in the age group above $60-65$ years was $26 \%$ and in the age group above 70 years was $40 \%$. Among these 53 patients (53\%) were Cerebral Thrombosis type, 31 patients (31\%) were cerebral haemorrhagic type, 6 patients $(6 \%)$ were of subarachnoid type and 10 patients $(10 \%)$ cerebral embolism type. Among 100 patients 52 were hypertensive, 28 were Diabetes mellitus patients.

In 100 cases studied 18 cases had normal ECG (18\%), while 82 cases $(82 \%)$ had some abnormality in the ECG. These abnormalities were commonly seen in cerebral haemorrhage and subarachnoid haemorrhage than infarcts. The ECG changes were QTc prolongation (30\%), ST Segment changes (28\%), T-wave changes (44\%), U-wave changes (10\%), LVH (36\%), sinus tachycardia (24\%), sinus bradycardia $(10 \%)$, Normal $(18 \%)$, a wave $(2 \%)$, sinus arrhythmia (2\%), VPC (2\%), LBBB (2\%), RBBB (2\%), P. pulmonale $(6 \%)$, atrial fibrillation is found in $(4 \%)$ of the patients. The overall immediate mortality was $22 \%$. It was $51.6 \%$ in cerebral haemorrhage, $33.34 \%$ in $\mathrm{SAH}, 20 \%$ in cerebral embolism and $3.7 \%$ was in cerebral thrombosis. These findings were similar to the Bozluolcay M (2003), Negh KH (2004), Fure B (2006) above mentioned studies.

\section{CONCLUSION}

Patients with cerebrovascular accidents often have abnormal electrocardiogram in the absence of known organic heart disease or electrolyte imbalance. These ECG changes are more common in intracerebral haemorrhage and subarachnoid haemorrhage than in infarcts. The common ECG changes are T-wave changes, prolonged Q-Tc, ST segment changes and arrhythmias. These changes are presumed to be due to alterations in sympathetic and parasympathetic tone. The mortality in these patients did not relate to the ECG changes seen, but was dependent on the type of cerebrovascular accident and the level of consciousness on admission. ECG changes are prevalent in acute ischaemic stroke. Atrial fibrillation was more frequent in ischaemic stroke than in haemorrhagic stroke. ST depression and $Q$ waves, suggesting that these ECG changes may indicate coexisting ischaemic heart disease. Regardless stroke-related lesion, ECG abnormalities can be seen frequently in stroke patients without primary heart disease. They can lead to diagnostic and therapeutic difficulties. The observations of this study suggest that cardiac evaluation in patients with acute ischaemic stroke is of prognostic importance. Patients with acute ischaemic stroke should be 
offered adequate treatment with secondary prevention and preferably a follow-up with focus on cardiologic as well as neurological aspects.

\section{REFERENCES}

1. Yamour BJ, Sridharan MR, Rice JF, et al. ECG changes in cerebrovascular haemorrhage. Am Heart J 1980;99(3):294-300.
2. Norris. Focal myocardial lesion in stroke, neurogenic cardiac effect of cerebrovascular disease. Curr Opin Neurol 1994;7(1):20-4.

3. Goldstein DS. ECG in stroke: relationship to pathophysiological type and comparison with prior tracing. Stroke 1979;10(3):253-9. 